\title{
Clinical characteristics and health care cost among patients successfully treated for COVID-19 in Henan, China: A Descriptive Study
}

\section{Yudong Miao}

Henan Provincial People's Hospital

Soumitra S Bhuyan

Rutgers University

Mengzhu Liu

Henan Provincial People's Hospital

Jianqin Gu ( $\nabla$ jiangpanzy@sina.com )

Henan Provincial People's Hospital

Sabiha Hussain

Rutgers University

Jian Wu

Zhengzhou University

Liuyi Wang

Henan Provincial People's Hospital

Min Liu

Henan Provincial People's Hospital

Ruirui Cheng

First Affilicated Hospital of Zhengzhou University

Urmi Basu

Insight Biopharma

Feng Liu

Henan Provincial People's Hospital

Huayu Zhang

Henan University

Zhanjun Chang

Zhengzhou Center for Disease Control and Prevention

\section{Research}

Keywords: COVID-19, health care cost, henan, medical expense 
Posted Date: June 8th, 2020

DOl: https://doi.org/10.21203/rs.3.rs-31030/v1

License: (c) (i) This work is licensed under a Creative Commons Attribution 4.0 International License. Read Full License 


\section{Clinical characteristics and health care cost among patients successfully treated for COVID-19 in}

\section{Henan, China: A Descriptive Study}

Yudong Miao*, PhD, Intelligent Hospital Engineering Research Center of Henan Provincial People's Hospital, People's Hospital of Zhengzhou University; Email: meldon1989@163.com

Soumitra S. Bhuyan*, PhD, Edward J Bloustein School of Planning and Public Policy, Rutgers University;

Email: soumitra.bhuyan@rutgers.edu

Mengzhu Liu*, Intelligent Hospital Engineering Research Center of Henan Provincial People's Hospital, People's Hospital of Zhengzhou University; Email: farioknow@sohu.com

Jianqin Gu, Professor, Intelligent Hospital Engineering Research Center of Henan Provincial People's Hospital, People's Hospital of Zhengzhou University; Email: jiangpanzy@ sina.com

Sabiha Hussain, MD, Division of Pulmonary and Critical Care, Robert Wood Johnson Medical School, Rutgers University; Email: hussaisa@rwjms.rutgers.edu

Jian Wu, Professor, College of Public Health, Zhengzhou University; Email: wujian@zzu.edu.cn

Liuyi Wang, Professor, General Medicine Department of Henan Provincial People's Hospital, People's

Hospital of Zhengzhou University; Email:Wb2007801 @ 126.com;

Min Liu, Professor, Hypertension Department of Henan Provincial People's Hospital, People's Hospital of Zhengzhou University; Email: conformiting@126.com

RuiRui Cheng, MD, Respiratory Department of the First Affiliated Hospital of Zhengzhou University; Email: Chengrui.nancy@163.com;

Urmi Basu, PhD, Insight Biopharma, New Jersey; Email: basurmi@gmail.com;

Zhanjun Chang, Professor, Zhengzhou Center for Disease Control and Prevention; Email:

langlangspring@sina.com;

Feng Liu, PhD, Public Health Medical Center, Intelligent Hospital Engineering Research Center of Henan Provincial People’s Hospital, People's Hospital of Zhengzhou University; Email: skyintcity@ 163.com Huayu Zhang, PhD, School of Nursing and Health of Henan University; Email: redpapering@ 163.com *Joint first authors

Correspondence to: Prof Jianqin Gu, Public Health Medical Center, Intelligent Hospital Engineering Research Center of Henan Provincial People's Hospital, People's Hospital of Zhengzhou University, Jinshui District, Zhengzhou 450003, China; Email: jiangpanzy@sina.com. 


\begin{abstract}
Objective: To clarify the clinical and medical expense characteristics of COVID-19.
\end{abstract}

Methods: In this retrospective, single-center study, 55 cured cases with confirmed COVID-19 were analyzed for demographic, epidemiological, clinical, and radiological features and medical expense data.

Results: The average age of the 54 successfully treated patients with COVID-19 was 53.2 years old (SD 19.0), including 27 men and 27 women. Off this, 31 (57.4\%) patients had chronic diseases. Patients commonly had clinical manifestations of fever (45 [83.3\%] patients), cough (29[54.7\%] patients), expectoration (28 [51.9\%] patients), fatigue (24[44.4\%] patients) and diarrhea (8[14.8\%] patients) on admission. There was a 10-day interval from the onset of signs and symptoms to hospital admission. About $80 \%$ of them got recovery after a two-week treatment. The mean interval from the onset of signs and symptoms to hospital discharge was 20.5 (IQR 16-29) days. The median total medical expense of the treated patient, in general, was 2579.6 (IQR 1366.1-4837.6) U.S. dollars. Still, the median medical expense was 8904.1 (IQR 6660.1- 27143.8) U.S. dollars in patients with more than five comorbid illnesses during the treatment.

Conclusion: There is a 3-week interval from the onset of signs and symptoms to cure, and most hospitalized patients get recovery within two weeks. The total medical expense of cases with more than five comorbid conditions during the treatment is higher. Quite a few COVID-19 cases with other serious diseases are likely to account for most of the total medical expenses. 


\section{Research in context}

\section{Evidence before this study}

We searched PubMed on May 13, 2020, for articles that describe the epidemiological, clinical, and health economic characteristics of COVID-19, using the search terms "COVID-19" or "2019 novel coronavirus (2019-nCoV)" with no language or time restrictions. Previously published research discussed the sources if SARS-CoV-2, epidemiological and clinical characteristics, mechanism, symptoms and diagnosis, prevention, and treatment strategies of COVID-19. The only report of the health care cost data of COVID-19 on China and the world was published on Apr 16, 2020, with analysis from the socioeconomic perspective. To the best of our knowledge, no study has examined the hospital cost data of COVID-19.

\section{Added value of this study}

This study is, to our knowledge, the earliest study to date of COVID-19 infections, with 54 cured patients admitted into Henan Provincial People's Hospital and provides further information on the demographic, clinical, epidemiological, and health care cost of patients. It presents the latest status of COVID-19 treatment and control in China and is an extended investigation of the previous report, with more details on health services utilization.

\section{Implications of all the available evidence}

There is a 3-week interval from the onset of signs and symptoms to cure, and most hospitalized patients get recovery within two weeks. COVID-19 infection does not cost much to treat for most cases, with quite a few cases with more than five comorbid diseases during the treatment accounting for most of the expenses. Priority should be given on timely hospitalization, and active intervention for comorbid conditions to effectively lower the medical costs and prevent the spread of this coronavirus in China and worldwide. 


\section{Introduction}

Since Dec 8, 2019, COVID-19 epidemic occurred in Wuhan, Hubei province, China. ${ }^{1-4}$ In the past months, COVID-19 has resulted in more than 3.6 million confirmed cases, and 250 thousand died cases globally, exceeding the impacts of confirmed cases of SARS (8273 cases, 775 deaths) and MERS (2494 cases and 858 deaths). ${ }^{5-7}$ COVID-19 infection has clinical symptoms of respiratory diseases resembling SARS, with fever, fatigue, dry cough as the primary manifestations. ${ }^{8-11}$ Most of the patients were moderate to mild, with a good prognosis, and a few were in critical condition. ${ }^{12,13}$ Based on the current epidemiological evidence, the incubation period of COVID-19 is 1-14 days, mostly 3-7 days. ${ }^{14-16}$ There is evidence of human-to-human transmission, and asymptomatic patients may also become the source of infection. ${ }^{17,18}$ The main transmission routes are respiratory droplet transmission and contact transmission. ${ }^{19}$ People of all ages are susceptible. ${ }^{20,21}$ Children show better prognosis than the elderly and adults with chronic diseases have poor. $^{22}$

At present, information regarding clinical characteristics and health care cost of patients successfully

treated for COVID-19 is scarce. ${ }^{23,24}$ In this study, we did a comprehensive exploration of the clinical and health economic features of 54 successfully treated patients with confirmed COVID-19 admitted to Henan Provincial People's Hospital, Zhengzhou, which cured all the patients with COVID-19 to be reported on.

\section{Methods}

\section{Study design and participants}

For this retrospective, single-center study, we recruited patients from Jan 23 to Feb 27, 2020, at Henan Provincial People's Hospital in Zhengzhou, China. Henan Provincial People's Hospital is a Regional Medical Center of central China with the Infectious Disease Department as its critical specialized department. As COVID-19 medical observation and hospitalization institution designated by the Chinese government, the hospital received all suspected patients with COVID-19 related symptoms (fever or respiratory symptoms) from the whole of Henan province and surrounding provinces without selectivity. All patients diagnosed as having COVID-19, according to World Health organization (WHO) interim 
guidance was enrolled in this study. ${ }^{25}$ All the data of included cases have been reported to provincial and national CDCs. No patients of this retrospective study were directly involved in the recruitment and design and identified through the anonymized database. The study was approved by the Henan Provincial People's Hospital Ethics Committee.

\section{Procedures}

The demographical, clinical, and health care cost data were obtained from the Hospital Information System of Henan Provincial People's Hospital. Clinical outcomes were followed up to the discharge date of the last patient (Mar 10, 2020). In terms of data missing from the records or clarification was needed, we obtained data by direct communication with healthcare providers of the Infectious Disease Department of Henan Provincial People's Hospital. There was no missing health economic data. All data were checked by three independent physicians (LM, LF, and CRR).

\section{Outcomes}

We described demographic characteristics (age, sex, marriage, etc.) and epidemiological characteristics (allergy drug, morbid chronic disease, exposure history, etc.); Clinical characteristics(signs and symptoms at admission, duration of Signs and symptoms at admission, comorbidities, etc.); Chest radiography and Computed Tomography (CT) findings; Treatment (Oxygen therapy, Mechanical ventilation, traditional Chinese medicine treatment, etc.) and outcome (cure rate); Health economic characteristics (total medical expenses and composition) and the correlations between total medical expense and comorbid chronic medical illness.

\section{Statistical analysis}

We presented continuous variables as mean (SD) and median (IQR), respectively, and categorical variables as count (\%). We used linear regression to analyze the correlation between the total medical expense and comorbid chronic medical illnesses among the sampled patients. $\mathrm{P}<0.05$ was considered statistically significant. Statistical analysis was performed using SPSS (Version 26.0).

\section{Role of the funding source}

The funder has no role in study design, analysis, and interpretation of the study findings. 


\section{Results}

A total of 54 patients with COVID-19 were included in this study, and each of them come from different families. The mean age for all patients was 54.2 years old, ranging from 20 to 90 years old, and patients aged 50-59 were most numerous (31.5\%). Half of the patients were male (50.0\%). 23 (42.6\%) patients lived in rural areas. Off these, 31(56.4\%) patients had chronic diseases, and ten (18.5\%) had more than one chronic disease. Five patients had a self-reported medical history of Penicillin or Piperacillin hypersensitivity (table 1).

On admission, the patients commonly experienced symptoms of fever (83.6\%), cough (55.6\%), expectoration (50.9\%), fatigue $24(44.4 \%)$, and diarrhea (14.5\%) (table 2). The mean interval from the onset of signs and symptoms to hospital admission was 10 (5-25) days. More than a third of the patients were admitted within one week, but $9(16.7 \%)$ had signs and symptoms for more than three weeks before seeking hospitalization services. Some patients presented with organ function damage, including 14 patients $(25.5 \%)$ with acute respiratory injury, seven patients $(12.7 \%)$ with ARDS, four patients (7.3\%) with septic shock, and three patients (5.5\%) with acute renal injury. According to chest X-ray and CT, 53 (98.2\%) patients showed bilateral pneumonia, with only $14(23.3 \%)$ patients showing unilateral pneumonia (table 2). It's worth noting that 38 (70.4\%) patients showed multiple mottling and groundglass opacity (table 2).

All patients were treated in isolation. All patients received antiviral treatment, and the duration of antiviral treatment was 5-14 days (median7.2 days [IQR 6-11]). Most patients (87.0\%) were given antibiotic treatment (table 2); 46(85.2\%) patients were given combination therapy. Only 1(1.9\%) patient was given CRRT and ECMO therapy. Also, 49 (90.7\%) patients were given proprietary Chinese medicine (including traditional Chinese medicine injection) recommended by the National Health Commission (table 2).

During the treatment, $43(78.2 \%)$ patients had at least one comorbid condition, $11(20.4 \%)$ had more than five comorbid illnesses. By the end of Mar 10, 2020, all patients had been discharged. The mean interval 
from the onset of signs and symptoms to hospital discharge was 20.5 (16-29) days. The median length of stay was 9.5 (6.8-12.3) days. About a quarter of the patients got discharged within one week, and patients receiving 1 to 2 weeks of hospitalization services were most numerous. The median total medical expense was 2579.6 (\$1366.1-4837.6) U.S dollar, comprising drug expenses (37.5\%), health services expenses (29.1\%), test expenses (18.1\%), and medical material expenses (15.3\%). The medical costs varied greatly among different patients. The 54 patients cost \$ 504663.2 U.S. dollars in total, with the top five highest expenses accounting for 70.2\% (\$354349.9 US dollars). The highest medical expense (US \$212542.6) came from a patient using a combination of ECMO and CRRT therapies. The intervals between the onset of symptoms and the discharge were 38 days (Jan 24 to Mar 10, 2020). The second-highest expense was \$ 51443.4 U.S. dollars, which was only less than a quarter of the highest cost.

Excluding the patient with the highest medical expense, the median medical cost was 8904.1 (IQR \$ 6660.1-27143.8) U.S dollars in patients with more than five comorbid conditions during the treatment, compared to the 2019.3 (IQR \$1231.2-\$3151.8) U.S. dollars in the rest of the patients $(\mathrm{P}<0.001$, picture A). The higher medical expense was found in organ function damage cases (median, \$6811.8 vs. \$1964.1, $\mathrm{P}<0.001$, picture $\mathrm{B})$. For patients who used CRRT and ECMO in the treatment, the medical expenses were significantly higher than the rest cases (median, $\$ 8904.1$ vs. $\$ 2163.6, \mathrm{P}<0.001$, picture C). The lowest cost was found in patients with a length of stay of less than one week (\$1433.9 vs. \$2908.2, $\mathrm{P}=0.008)$. No difference was identified for medical expenses among the rest patients with similar length of stay (picture D).

\section{Discussion}

This is an extended descriptive study on the clinical, epidemiological, and health care cost of 54 patients successfully treated patients with COVID-19, who were hospitalized in Henan Provincial People's Hospital. As a pandemic disease identified by WHO, COVID-19 is resulting in more death with an estimated case fatality rate ranging from $0.15 \%$ to $11 \% .{ }^{26-29}$ At data cut off for this study, no case of the 54 included patients infected by COVID-19 died. However, additional deaths might occur in subsequent 
cases. As new reports of re-infections among previously treated COVID-19 cases come from some countries, including China, the health status of the discharged patients' needs continuous follow-up. In the present study, we found that most patients were middle- and old-aged, with 1:1 male-female ratio. The mean age (53.2[SD 19.0])) is younger than data reported by Chen et al. and Zhang et al. ${ }^{29,30} 56.4 \%$ of the patients had at least one chronic illness on admission. During the treatment, $78.2 \%$ of them developed comorbid conditions, many of them progressed into organ dysfunction, including acute respiratory injury (ARDS), septic shock, and acute renal injury. Therefore, early identification and timely treatment of critical cases are crucial for preventing disease and multiorgan failure. Although fever (83.6\%), cough (55.6\%), expectoration (50.9\%), fatigue (44.4\%) and diarrhea (14.5\%) were the most common symptoms in the 54 patients, the prevalence of symptoms in this cohort was significantly lower than previously reported data. ${ }^{21,31}$ This might be one of the reasons why all 54 patients were cured, it is known that fever severity correlates with disease severity and risk of developing ARDS. ${ }^{32}$ These individuals where many were asymptomatic carriers or that the disease may represent a cohort of mild infection. They also were caught early and treated. It's important to note that there was a 10-day interval from the onset of signs and symptoms to hospital admission. These asymptomatic or mild patients may cause more infections during the interval. Considering the human-to-human transmission characteristics and the unknown transmission rate of COVID-19, it is necessary to reduce the population's mobility during the pandemic.

In terms of treatment, minimizing treatment duration and comorbidities are critical for patient management. Most hospitalized patients were given treatments according to the COVID-19 protocol recommended by Chinese National Health Commission (version 1 was published on Jan 23, 2020, the latest version 7 was released on Mar 4, 2020), including oxygen therapy, antibiotics, antivirals, Glucocorticoids, and traditional Chinese medicine. Considering that old age and comorbidities might be associated with low immunity and increased mortality, many of them were given a combination of antibiotics and intravenous immunoglobulin to prevent infection and strengthen their immune system. For patients with ARDS, steroids (methylprednisolone $1-2 \mathrm{mg} / \mathrm{kg}$ per day) were used for as short a duration 
of treatment as possible. In general, the treatments were effective, with all patients cured, and about $80 \%$ of them got recovery within the two-weeks treatment duration.

According to our data, COVID-19 is not very expensive to treat (Median 2579.6 [IQR 1366.1-4837.6]), compared to the average cost of tertiary hospitalization of China in 2018 (US $\$ 1885.7)^{33}$ and Henan Provincial People's Hospital in 2019(\$2971.5). During treatment, if individuals developed more than five comorbid diseases in patients, their total medical expenses are likely to increase significantly. Further, although the sequelae of COVID-19 are not entirely clear, patients with more than five comorbidities during the treatment are likely to have higher long-term health expenditure. Although CRRT combined with ECMO is useful in treating severe patients, the therapy is greatly costly. ${ }^{34,35}$ Timely intervention in patients and the prevention of progression to other comorbidities is crucial to reduce high medical costs. This study has several limitations. First, only 54 successfully treated patients with confirmed COVID-19 were included; suspected but undiagnosed cases were ruled out in the analyses. It would be better to include as many treated patients as possible in Zhengzhou, in other cities in China, and even in other countries to get a more comprehensive understanding of COVID-19 from a health economic perspective. Further, the true economic burden may not have been assessed since only four or only $7.4 \%$ of the patients were intubated in this cohort, whereas in the Wuhan, China cohort was close to $43 \% .{ }^{32,36}$ Future studies should examine the burden of COVID-19, particularly regarding health-adjusted life years, disability-adjusted life years, health-related quality of life, and potential years of life loss.

\section{Declarations}

\section{Ethics approval and consent to participate}

All enrolled patients gave written informed consent before the clinical treatment in Henan Provincial People's Hospital. The ethics committee of Henan Provincial People's Hospital ruled that no formal ethics approval was required in this particular case.

\section{Consent for publication}

All authors have approved the final manuscript and consented for the publication.

\section{Availability of data and material}


The anonymized dataset of this article is available through the email of the corresponding author.

\section{Competing interests}

The authors declare that there is no conflict of interest.

\section{Funding}

The study is sponsored by National Natural Science Foundation of China (number 71603132), and Special Fund for the Development of Local Science and Technology Guided by the Chinese Central Government (number Z135050009017).

\section{Authors' contributions}

YD M, MZ L, F L and HY Z collected the epidemiological and clinical data and processed statistical data. YD M, JQ G and ZJ C drafted the manuscript. S B, S H, U B, J W and RR C revised the final manuscript. LY W and M L are responsible for summarizing all data related to clinical features. YD M, JQ $\mathrm{G}$ and $\mathrm{HY} \mathrm{Z}$ are responsible for summarizing all health services utilization data.

\section{Acknowledgement}

The authors acknowledge the engineers of Wuhan Puro Solution Health Technology Co., Ltd. for extracting the data from Hospital Information System.

\section{References}

1 Zhou F, Yu T, Du R, et al. Clinical course and risk factors for mortality of adult inpatients with COVID-19 in Wuhan, China: a retrospective cohort study. Lancet 2020; 395(10229): 1054-1062.

2 Qin C, Zhou L, Hu Z, et al. Dysregulation of immune response in patients with COVID-19 in Wuhan, China. Clin Infect Dis 2020; ciaa248.

3 Shi H, Han X, Jiang N, et al. Radiological findings from 81 patients with COVID-19 pneumonia in Wuhan, China: a descriptive study. Lancet Infect Dis 2020; 20(4): 425-434.

4 Peeri NC, Shrestha N, Rahman MS, et al. The SARS, MERS and novel coronavirus (COVID-19) epidemics, the newest and biggest global health threats: what lessons have we learned? Int $J$ Epidemiol 2020; dyaa033.

5 Velavan TP, Meyer CG. The COVID-19 epidemic. Trop Med Int Health 2020; 25(3): 278-280. 
6 Mahase E. Coronavirus covid-19 has killed more people than SARS and MERS combined, despite lower case fatality rate. BMJ 2020; 368: m641.

7 Wang Y, Wang Y, Chen Y, et al. Unique epidemiological and clinical features of the emerging 2019 novel coronavirus pneumonia (COVID-19) implicate special control measures. J Med Virol 2020; 10.1002/jmv.25748.

8 Chen $\mathrm{H}$, Guo J, Wang $\mathrm{C}$, et al. Clinical characteristics and intrauterine vertical transmission potential of COVID-19 infection in nine pregnant women: a retrospective review of medical records. Lancet 2020; 395(10226): 809-815.

9 Zu ZY, Jiang MD, Xu PP, et al. Coronavirus Disease 2019 (COVID-19): A Perspective from China. Radiology 2020; 200490.

10 Guo YR, Cao QD, Hong ZS, et al. The origin, transmission and clinical therapies on coronavirus disease 2019 (COVID-19) outbreak - an update on the status. Mil Med Res 2020; 7(1):11.

11 Jiang F, Deng L, Zhang L, et al, Cheung CW, Xia Z. Review of the Clinical Characteristics of Coronavirus Disease 2019 (COVID-19). J Gen Intern Med 2020; 10.1007/s11606-020-05762-w.

12 Ludvigsson JF. Systematic review of COVID-19 in children shows milder cases and a better prognosis than adults. Acta Paediatr 2020; 10.1111/apa.15270.

13 Zhou F, Yu T, Du R, et al. Clinical course and risk factors for mortality of adult inpatients with COVID-19 in Wuhan, China: a retrospective cohort study. Lancet 2020; 395(10229): 1054-1062.

14 Lauer SA, Grantz KH, Bi Q, et al. The Incubation Period of Coronavirus Disease 2019 (COVID-19) From Publicly Reported Confirmed Cases: Estimation and Application. Ann Intern Med 2020; M20-0504.

15 Lai CC, Shih TP, Ko WC, et al. Severe acute respiratory syndrome coronavirus 2 (SARS-CoV-2) and coronavirus disease-2019 (COVID-19): The epidemic and the challenges. Int J Antimicrob Agents 2020; 55(3): 105924. 
16 Linton NM, Kobayashi T, Yang Y, et al. Incubation Period and Other Epidemiological

Characteristics of 2019 Novel Coronavirus Infections with Right Truncation: A Statistical Analysis of Publicly Available Case Data. J Clin Med 2020; 9(2): 538.

17 Bai Y, Yao L, Wei T, et al. Presumed Asymptomatic Carrier Transmission of COVID-19. JAMA 2020; e202565.

18 Wilder-Smith A, Chiew CJ, Lee VJ. Can we contain the COVID-19 outbreak with the same measures as for SARS? Lancet Infect Dis 2020; S1473-3099(20)30129-8.

19 Peng X, Xu X, Li Y, et al. Transmission routes of 2019-nCoV and controls in dental practice. Int J Oral Sci 2020; 12(1): 9.

20 Qin C, Zhou L, Hu Z, et al. Dysregulation of immune response in patients with COVID-19 in Wuhan, China. Clin Infect Dis 2020; ciaa248.

21 Liu W, Zhang Q, Chen J, et al. Detection of Covid-19 in Children in Early January 2020 in Wuhan, China. N Engl J Med 2020; 10.1056/NEJMc2003717.

22 Ludvigsson JF. Systematic review of COVID-19 in children shows milder cases and a better prognosis than adults. Acta Paediatr 2020; 10.1111/apa.15270.

23 Ayittey FK, Ayittey MK, Chiwero NB, et al. Economic impacts of Wuhan 2019-nCoV on China and the world. J Med Virol 2020; 92(5): 473-475.

24 Li LQ, Huang T, Wang YQ, et al. COVID-19 patients' clinical characteristics, discharge rate, and fatality rate of meta-analysis. J Med Virol 2020;10.1002/jmv.25757.

25 WHO. Clinical management of severe acute respiratory infection when Novel coronavirus (nCoV) infection is suspected: interim guidance. Jan 11, 2020. https://www.who.int/internalpublications-detail/clinical-management-of-severe-acute-respiratoryinfection-when-novel-coronavirus-(ncov)-infection-is-suspected (accessed Jan 20, 2020).

26 Onder G, Rezza G, Brusaferro S. Case-Fatality Rate and Characteristics of Patients Dying in Relation to COVID-19 in Italy. JAMA 2020; 10.1001/jama.2020.4683. 
27 Yang S, Cao P, Du P, et al. Early estimation of the case fatality rate of COVID-19 in mainland China: a data-driven analysis. Ann Transl Med 2020; 8(4): 128.

28 Porcheddu R, Serra C, Kelvin D, et al. Similarity in Case Fatality Rates (CFR) of COVID-19/SARSCOV-2 in Italy and China. J Infect Dev Ctries 2020; 14(2): 125-128.

29 Chen N, Zhou M, Dong X, et al. Epidemiological and clinical characteristics of 99 cases of 2019 novel coronavirus pneumonia in Wuhan, China: a descriptive study. Lancet 2020; 395(10223): $507-513$.

30 Zhang JJ, Dong X, Cao YY, et al. Clinical characteristics of 140 patients infected with SARS-CoV-2 in Wuhan, China. Allergy 2020; 10.1111/all.14238.

$31 \mathrm{Wu} \mathrm{F}$, Zhao S, Yu B, et al. A new coronavirus associated with human respiratory disease in China. Nature 2020; 579(7798): 265-269.

32 Wu C, Chen X, Cai Y, et al. Risk Factors Associated With Acute Respiratory Distress Syndrome and Death in Patients With Coronavirus Disease 2019 Pneumonia in Wuhan, China. JAMA Intern Med 2020; e200994.

33 National Health Commission. China Health Statistics Yearbook 2019 [M]. China Union Medical University Press, Beijing: 2020.

34 Ramanathan K, Antognini D, Combes A, et al. Planning and provision of ECMO services for severe ARDS during the COVID-19 pandemic and other outbreaks of emerging infectious diseases. Lancet Respir Med 2020; S2213-2600(20)30121-1.

35 Fu D, Yang B, Xu J, Mao Z, et al. COVID-19 Infection in a Patient with End-Stage Kidney Disease. Nephron 2020; $1-3$.

36 Wang D, Hu B, Hu C, et al. Clinical Characteristics of 138 Hospitalized Patients With 2019 Novel Coronavirus-Infected Pneumonia in Wuhan, China. JAMA 2020; e201585. 
Table 1: Demographic information and Epidemiological characteristics of 55 successfully treated patients admitted to Henan Provincial People's Hospital (Jan 23- Mar 10, 2020) with COVID-19

\begin{tabular}{ll}
\hline & Patients $\mathbf{( n = 5 4 )}$ \\
\hline Age, years & \\
Mean (SD) & $53.2(19.0)$ \\
Range & $20-90$ \\
$\leq 29$ & $9(16.7 \%)$ \\
$30-39$ & $6(11.1 \%)$ \\
$40-49$ & $5(9.3 \%)$ \\
$50-59$ & $17(31.5 \%)$ \\
$60-69$ & $6(11.1 \%)$ \\
$70-79$ & $6(11.1 \%)$ \\
280 & $5(9.3 \%)$ \\
SEX & \\
Female & $27(50.0 \%)$ \\
Male & $27(50.0 \%)$ \\
Place of residence & \\
Rural & $23(42.6 \%)$ \\
Urban & $31(57.4 \%)$ \\
Health insurance & \\
Social health insurance & $37(68.5 \%)$ \\
Commercial medical insurance & $12(22.2 \%)$ \\
No health insurance & $5(9.3 \%)$ \\
Allergy Drug & \\
None & $49(81.6 \%)$ \\
Penicillin & $4(6.7 \%)$ \\
Piperacillin & $1(1.7 \%)$ \\
Chronic illness & \\
Any & $31(57.4 \%)$ \\
0 disease & $23(42.6 \%)$ \\
1 disease & $21(38.8 \%)$ \\
2 diseases & $5(9.3 \%)$ \\
3 diseases & $3(5.6 \%)$ \\
4 diseases & $2(3.7 \%)$ \\
\hline Data a & \\
\hline
\end{tabular}

Note: Data are n (\%); COVID-19= Corona Virus Disease 2019. SD=standard deviation. Three medical staffs were diagnosed with COVID-19, but none of them was the staff of Henan Provincial People's Hospital. 
Table 2: Clinical characteristics, treatment and outcome of patients with COVID-19

Patients $(n=54)$

\begin{tabular}{|c|c|}
\hline \multicolumn{2}{|l|}{ Signs and symptoms at admission } \\
\hline Fever & $45(83.3 \%)$ \\
\hline Cough & $29(54.7 \%)$ \\
\hline Expectoration & $28(51.9 \%)$ \\
\hline Fatigue & $24(44.4 \%)$ \\
\hline Diarrhoea & $8(14.8 \%)$ \\
\hline \multicolumn{2}{|l|}{ Onset of symptoms to hospital admission, Day } \\
\hline Median (IQR) & $10.0(5-20)$ \\
\hline$\leq 7$ & $19(35.2 \%)$ \\
\hline $8-14$ & $16(29.6 \%)$ \\
\hline $14-20$ & $10(18.5 \%)$ \\
\hline$\geq 21$ & $9(16.7 \%)$ \\
\hline \multicolumn{2}{|l|}{ Chest X-ray and CT findings } \\
\hline Unilateral pneumonia & $14(23.3 \%)$ \\
\hline Bilateral pneumonia & $53(98.1 \%)$ \\
\hline Multiple mottling and ground-glass opacity & $38(70.4 \%)$ \\
\hline \multicolumn{2}{|l|}{ Treatment } \\
\hline Oxygen therapy & $41(74.5 \%)$ \\
\hline \multicolumn{2}{|l|}{ Mechanical ventilation } \\
\hline Non-invasive (ie, face mask) & $11(20.4 \%)$ \\
\hline Invasive & $4(7.4 \%)$ \\
\hline CRRT & $8(14.8 \%)$ \\
\hline ECMO & $2(3.7 \%)$ \\
\hline Antibiotic treatment & $47(87.0 \%)$ \\
\hline Antifungal treatment & $7(13.0 \%)$ \\
\hline Antiviral treatment & $54(100.0 \%)$ \\
\hline Glucocorticoids & $37(68.5 \%)$ \\
\hline Intravenous immunoglobulin therapy & $9(16.7 \%)$ \\
\hline Traditional Chinese medicine treatment & $49(90.7 \%)$ \\
\hline \multicolumn{2}{|l|}{ Comorbid illness during the treatment } \\
\hline Any & $43(78.2 \%)$ \\
\hline 0 disease & $12(22.2 \%)$ \\
\hline 1 disease & $13(24.1 \%)$ \\
\hline 2 diseases & $7(13.0 \%)$ \\
\hline 3 diseases & $6(11.1 \%)$ \\
\hline 4 diseases & $5(9.3 \%)$ \\
\hline$\geq 5$ diseases & $11(20.4 \%)$ \\
\hline \multicolumn{2}{|l|}{ Organ function damage } \\
\hline Acute respiratory injury & $13(24.1 \%)$ \\
\hline ARDS & $7(13.0 \%)$ \\
\hline Septic shock & $4(7.4 \%)$ \\
\hline Acute renal injury & $3(5.6 \%)$ \\
\hline \multicolumn{2}{|l|}{ Clinical outcome } \\
\hline Remained in hospital & $0(100.0 \%)$ \\
\hline Discharged & $54(100.0 \%)$ \\
\hline Died & $0(100.0 \%)$ \\
\hline \multicolumn{2}{|l|}{ Length of stay, Day } \\
\hline Median (IQR) & $9.5(6.8-12.3)$ \\
\hline$\leq 7$ & $14(25.9 \%)$ \\
\hline $8-13$ & $29(53.7 \%)$ \\
\hline $14-20$ & $7(13.0 \%)$ \\
\hline
\end{tabular}


$\geq 21$

Onset of symptoms to Discharge (Day)

Median (IQR)

$\leq 13$

$14-20$

$\geq 21$

Note: IQR=interquartile range. $A R D S=$ acute respiratory distress syndrome. $E C M O=$ extracorporeal membrane oxygenation. CRRT=continuous renal replacement therapy. COVID-19= Corona Virus Disease 2019.
$4(7.4 \%)$

20.5(16-29)

$7(13.0 \%)$

$20(37.0 \%)$

$27(50.0 \%)$ 
Table 3: Medical expense characteristics of successfully treated patients with COVID-19

\begin{tabular}{ll}
\hline & Patients $(\mathbf{n}=\mathbf{5 4})$ \\
\hline Medical expense (\$)-median (IQR) & \\
Total medical expense & $2579.6(1366.1-4837.6,100 \%)$ \\
Drug expense & $705.5(386.0-705.5,37.5 \%)$ \\
Health services expense & $647.4(407.0-1256.5,29.1 \%)$ \\
Test expense & $932.6(576.4-932.6,18.1 \%)$ \\
Medical material expense & $76.2(38.5-177.9,15.3 \%)$ \\
Distribution of total medical expense & \\
Top 1 case & $212542.6(42.1 \%)$ \\
Top 5 cases & $354349.8(70.2 \%)$ \\
Top 15 cases & $424716.1(84.2 \%)$ \\
Top 25 cases & $459592.7(91.1 \%)$ \\
Top 35 cases & $482164.8(95.5 \%)$ \\
Top 45 cases & $496109.4(98.3 \%)$ \\
All 54 cases e throat & $504663.2(100.0 \%)$ \\
\hline
\end{tabular}

Note: Data are $\mathrm{n}(\%), \mathrm{n} / \mathrm{N}(\%)$, and median (IQR). COVID-19= Corona Virus Disease 2019. The exchange rate between RMB and US dollar is 1 to 7.095 . 

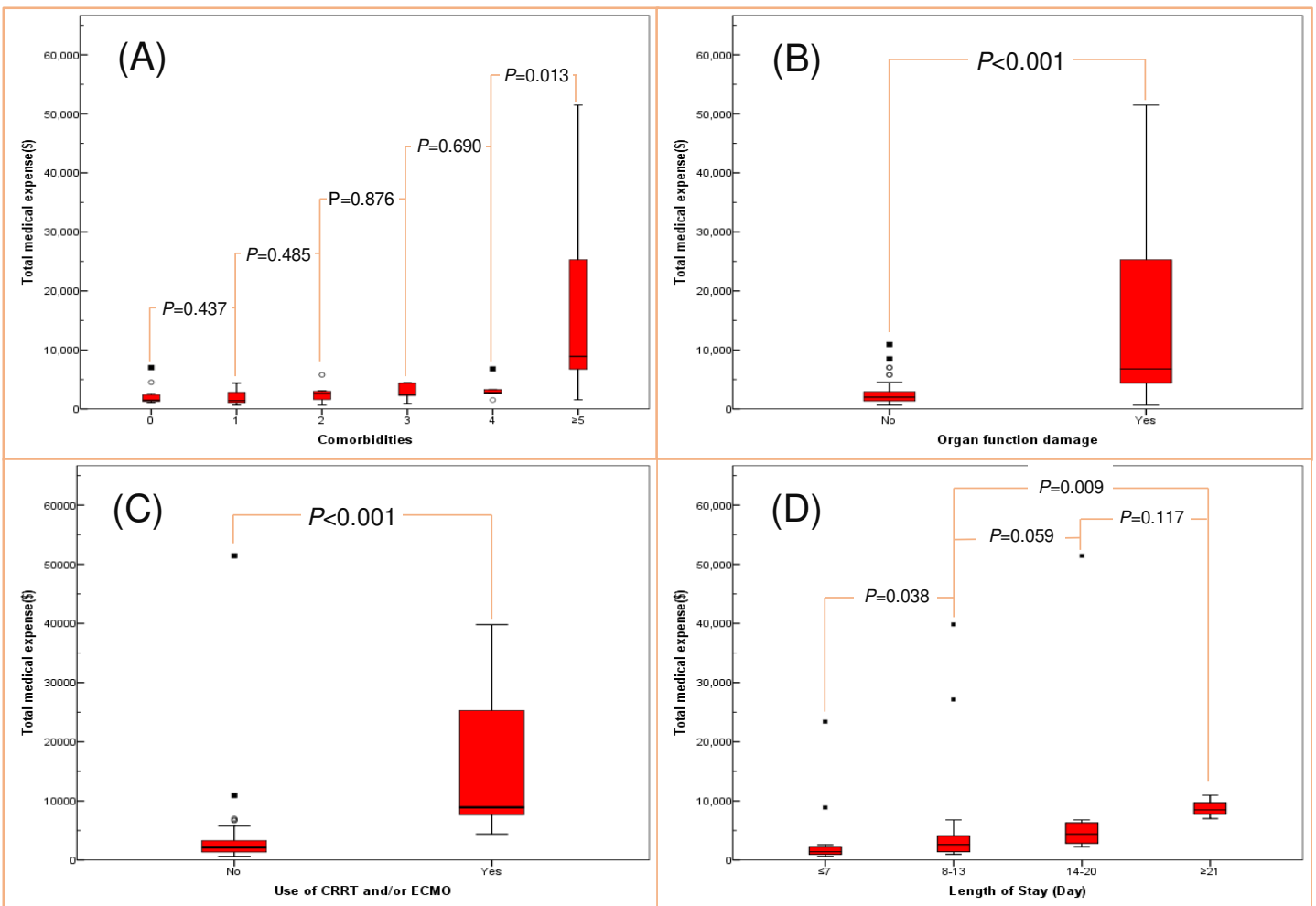

Figure: Comparisons of total medical expenses between/among different groups of 53 successfully treated patients with COVID-19.

Figure A-D did not contain the medical expense of the patient with the highest medical expenses $(\$ 212542.6$, accounting for $42.1 \%$ of the total medical expenses of the 54 patients). 
Figures

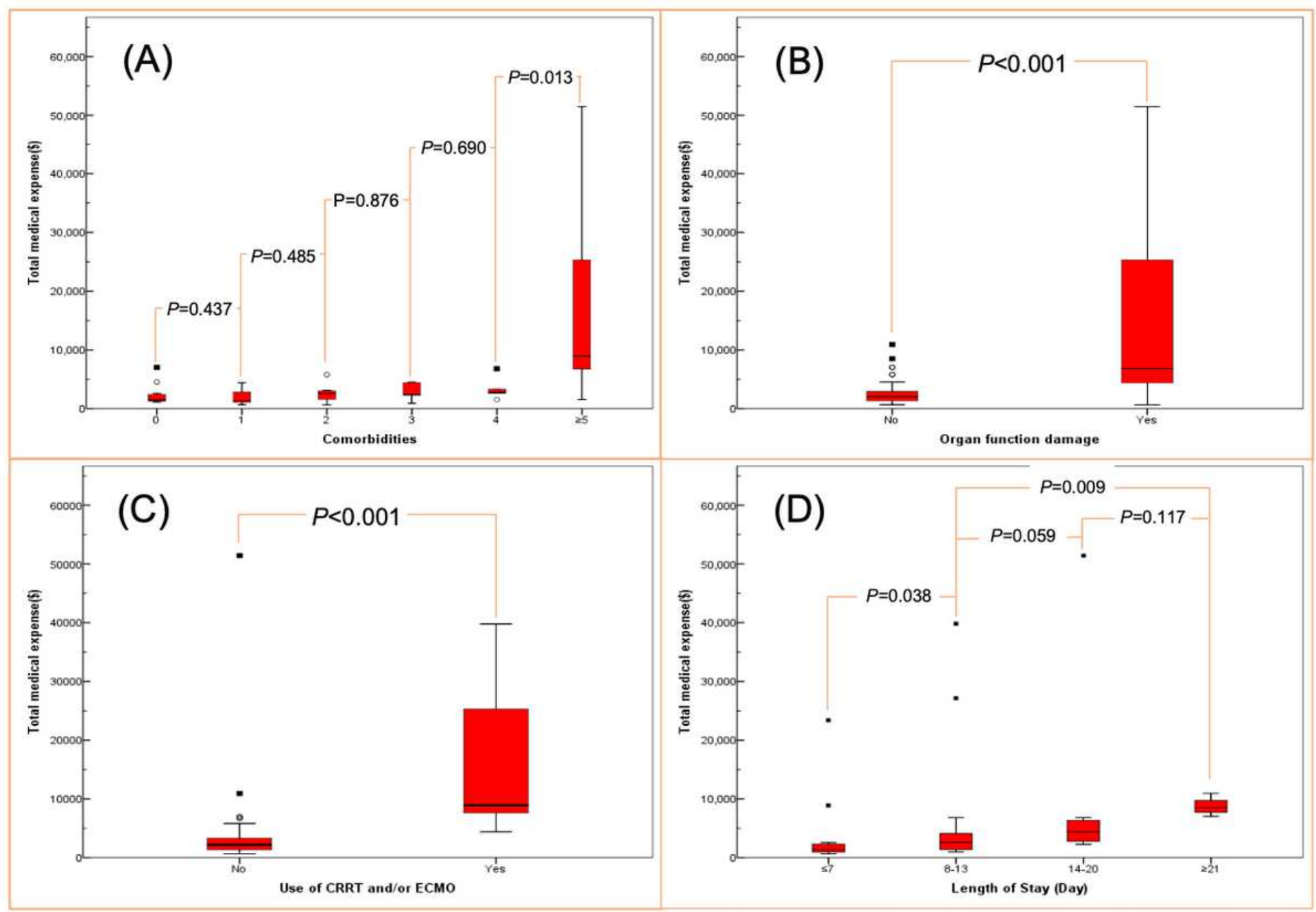

Figure 1

Comparisons of total medical expenses between/among different groups of 53 successfully treated patients with COVID-19. Figure A-D did not contain the medical expense of the patient with the highest medical expenses ( $\$ 212542.6$, accounting for $42.1 \%$ of the total medical expenses of the 54 patients). 